\title{
Overexpression of CDC25B, CDC25C and phospho-CDC25C (Ser216) in vulvar squamous cell carcinomas are associated with malignant features and aggressive cancer phenotypes
}

\author{
Zhihui Wang1,2, Claes G Trope³, Vivi Ann Flørenes, Zhenhe Suo', Jahn M Nesland¹ and Ruth Holm4
}

\begin{abstract}
Background: CDC25 phosphatases are important regulators of the cell cycle. Their abnormal expression detected in a number of tumors implies that their dysregulation is involved in malignant transformation. However, the role of CDC25s in vulvar cancer is still unknown. To shed light on their roles in the pathogenesis and to clarify their prognostic values, expression of CDC25A, CDC25B and CDC25C in a large series of vulvar squamous cell carcinomas were examined.

Methods: Expression of CDC25A, CDC25B, CDC25C and phosphorylated (phospho)-CDC25C (Ser216) were examined in 300 vulvar carcinomas using immunohistochemistry. Western blot analysis was utilized to demonstrate CDC25s expression in vulvar cancer cell lines. Kinase and phosphatase assays were performed to exclude cross reactivity among CDC25s isoform antibodies.

Results: High nuclear CDC25A and CDC25B expression were observed in $51 \%$ and $16 \%$ of the vulvar carcinomas, respectively, whereas high cytoplasmic CDC25C expression was seen in 63\% of the cases. In cytoplasm, nucleus and cytoplasm/nucleus high phospho-CDC25C (Ser216) expression was identified in 50\%, 70\% and 77\% of the carcinomas, respectively. High expression of CDC25s correlated significantly with malignant features, including poor differentiation and infiltration of vessel for CDC25B, high FIGO stage, presence of lymph node metastases, large tumor diameter, poor differentiation for CDC25C and high FIGO stage, large tumor diameter, deep invasion and poor differentiation for phospho-CDC25C (Ser216). In univariate analysis, high expression of phospho-CDC25C (Ser216) was correlated with poor disease-specific survival $(p=0.04)$. However, such an association was annulled in multivariate analysis.

Conclusions: Our results suggest that CDC25C and phospho-CDC25C (Ser216) play a crucial role and CDC25B a minor role in the pathogenesis and/or progression of vulvar carcinomas. CDC25B, CDC25C and phospho-CDC25C (Ser216) were associated with malignant features and aggressive cancer phenotypes. However, the CDC25s isoforms were not independently correlated to prognosis.
\end{abstract}

\section{Background}

Vulvar carcinoma, counting for 3-5\% of all female genital cancers [1], is a disease most frequently observed in elder women. However, recently an increase in its incidence was also seen among younger women [2,3]. Although surgery is still kept as the standard treatment [3], considerable morbidity is often inevitably raised as a consequence of radical surgery [4]. In a bid to decrease the incidence of

* Correspondence: ruth.holm@radiumhospitalet.no

${ }^{4}$ Department of Pathology, Oslo University Hospital, Norway Full list of author information is available at the end of the article complications, there has been a movement towards individualized therapy and less radical surgery. In this sense, knowledge of biomolecular markers would be of considerable value to yield a better treatment decision.

CDC25 phosphatases, which are believed to be important regulators of cell cycle progression, dominate entry into mitosis by regulating the activation of CDK1/cyclin B [5]. Catalyzed by these dual specificity phosphatases, cyclin/CDKs are dephosphorylated and actived after removal of inhibitory phosphate groups from $\mathrm{Thr}^{14}$ and

() 2010 Wang et al; licensee BioMed Central Ltd. This is an Open Access article distributed under the terms of the Creative Commons B. Wed Central Attribution License (http://creativecommons.org/licenses/by/2.0), which permits unrestricted use, distribution, and reproduction in any medium, provided the original work is properly cited. 
Tyr ${ }^{15}$ [6]. In human, three isoforms of CDC25 denoted CDC25A, CDC25B and CDC25C exist. Initially, $\mathrm{CDC} 25 \mathrm{~A}$ is found to act at the G1/S transition, whereas $\mathrm{CDC} 25 \mathrm{~B}$ and CDC25C mainly play their roles at the G2/ $M$ transition [5,7]. However, recent studies suggest that all three $\mathrm{CDC} 25$ phosphatases function as regulators of both $\mathrm{G} 1 / \mathrm{S}$ and $\mathrm{G} 2 / \mathrm{M}$ transitions [7].

Although exact reasons of tumorigenesis remain unknown, it is believed that one of the hallmarks of tumorigenesis is dysregulation of cell proliferation, and thus is strongly suggested to be connected with disorders of cell cycle [6,8-10]. CDC25s are implied to be involved in the malignant transformation when deficient checkpoints are performed during mitosis $[6,11]$. The activity of the CDC25s are regulated by their phosphorylation status, expression level and subcellular localization [6,11]. Previously, abnormal expression of CDC25s have been reported in a number of carcinomas, such as breast [12], ovarian [13], esophageal [14], prostate [15] and colorectal carcinomas [9]. Overexpression of CDC25 isoforms are supposed to contribute to tumorigenesis by enhancing tumor malignancy [5]. To our knowledge, expression of CDC25s in vulvar cancers has not yet been reported. The aims of our study were to determine expression statuses of CDC25A, CDC25B and CDC25C in a large series of vulvar squamous cell carcinomas to shed light on their roles in the pathogenesis of this cancer type and to clarify their potential prognostic values.

\section{Methods}

\section{Patient materials}

A retrospective study including 300 cases of vulvar squamous cell carcinoma was performed. These patients underwent resection at The Norwegian Radium Hospital from 1977 to 2006. The median age at diagnosis was 74 years (range 35-96 years). Pre-surgery treatment was given to 9 patients, of which 6 received radiotherapy, whereas the other 3 were treated with radiotherapy/chemotherapy. Two hundred and one (67\%) patients received radical vulvectomy. Postoperative treatment including irradiation, chemotherapy and irradiation/chemotherapy were performed on $63(21 \%), 3(1 \%)$, and $4(1 \%)$ of the patients, respectively. Relapse was observed in 107 (36\%) patients. All patients were followed up since confirmed diagnosis until death or 31. December, 2006. One hundred and twenty (40\%) patients died of vulvar cancer. The median follow-up time for patients still alive was 131 months (range 11 to 346 months). All tumors were staged based on the International Federation of Gynecology and the Obstetrics (FIGO) classification [16]. Approval of the study has been given by The Regional Committee for Medical Research Ethics South of Norway (S-06012), The Social- and Health Directorate (04/2639 and 06/1478) and The Data Inspectorate (04/01043).
Histological specimens were reviewed by J.M.N, one of the co-author, who was concealed from all clinical information. Classification was performed according to World Health Organization recommendations [17]. Two hundred and eighty-two (94\%) tumors were keratinizing/ nonkeratinizing, $14(5 \%)$ were basaloid and $4(1 \%)$ were veruccoid. Previously, we have examined the expression of 14-3-3 $\sigma$ and human papillomavirus (HPV) infection in primary vulvar carcinomas $[18,19]$, which was compared with the expression of CDC25s from the present study. Ten samples of normal vulva form patients undergoing surgery for benign gynecological diseases were included as control.

\section{Cell Line}

Two human vulvar squamous cell carcinoma cell lines, SW-954 (ATCC, Manassas, VA, USA) and CAL-39 (DSMZ, Germany), were cultured in RPMI 1640 medium (BioWhit-taker Europe, Verviers, Belgium) supplemented with $5 \%$ fetal bovine serum (FBS) (Biochrom KG, Berlin, Germany). For Western blot analysis and immunohistochemistry, monolayer cells were harvested by $0.01 \mathrm{M}$ EDTA and thereafter washed in PBS.

\section{Immunohistochemical method}

Four- $\mu \mathrm{m}$ sections made from formalin-fixed, paraffinembedded tissues and cell lines were immunostained using the Advance ${ }^{\mathrm{Tt}}$ HRP System (K4068, Dako Corporation, CA, USA). After deparaffinization, sections for CDC25A staining were microwaved in $10 \mathrm{mM}$ Tris-1 mM EDTA, pH 9.0, sections for CDC25B and phosphoCDC25C (Ser 216) staining were microwaved in $1 \mathrm{mM}$ EDTA, $\mathrm{pH} 8.0$ and sections for CDC25C staining were microwaved in $10 \mathrm{mM}$ citrate buffer, $\mathrm{pH} 6.0$ to regain the epitopes blocked by formalin fixation. To block endogeneous peroxidase the sections were treated with $0.3 \%$ hydrogen peroxide $\left(\mathrm{H}_{2} \mathrm{O}_{2}\right)$ for $5 \mathrm{~min}$. Sections were incubated overnight at $4^{\circ} \mathrm{C}$ with monoclonal antibodies, including CDC25A (clone DCS-120+DCS-121, 1:500, 0.4 $\mu \mathrm{g} \mathrm{IgG} \mathrm{Ia}_{2 \mathrm{a}} / \mathrm{ml}$ ), CDC25B (clone 25B03, 1:150, $1.3 \mu \mathrm{g} \mathrm{IgG} /$ $\mathrm{ml}$ ), CDC25C (clone 25C07, 1:100, $2 \mu \mathrm{g} \mathrm{IgG} / \mathrm{ml}$ ), all from NeoMarkers, CA, USA, and phospho-CDC25C (Ser 216) (clone 63F9, 1:500), from Cell Signaling, MA, USA. The specimens were then given a sequential $30 \mathrm{~min}$ incubation with Advance ${ }^{\mathrm{Tm}}$ HRP link and Adance HRP enzyme, followed by treatment with 3'3-diaminobenzidine tetrahydrochloride (DAB) for $10 \mathrm{~min}$, counterstained with hematoxylin, dehydrated and mounted in Diatex.

Sections from tonsil with known CDC25A, CDC25B and phospho-CDC25C (Ser 216) expression and from breast carcinoma with known CDC25C expression were used as positive control. Negative control included i) substitution of the monoclonal antibody with mouse myceloma protein of the same subclass and concentration as 
the monoclonal antibody, ii) incubation of sections with phospho-CDC25C (Ser 216) absorbed with phosphoCDC25C (Ser 216) peptide (Cell Signaling, MA, USA) as recommended by the supplier.

Semiquantitative classes were used to describe the intensity (absent, 0; weak, 1; moderate, 2; strong, 3) and extent of staining (percent of positive tumor cells: absent, $0 ;<10 \%, 1 ; 10-50 \%, 2 ;>50 \%, 3)$. By multiplying intensity score with extent score, product scores for both cytoplasm staining and nucleus staining were produced which ranging from 0 to 9 . By taking product scores from cytoplasm and nucleus into account at the same time, a composed score was given for each section. Based on staining pattern observed in normal vulvar epithelium, cutoff values in cytoplasm and/or nucleus were set. High CDC25A and $\mathrm{CDC} 25 \mathrm{~B}$ immunostaining in the nucleus was classified with a score $>6$, and low with a score $\leq 6$, whereas, high CDC25C and phospho-CDC25C (Ser 216) immunostaining in cytoplasm was classified with a score $>3$ and low with a score $\leq 3$. In addition, high phosphoCDC25C (Ser 216) immunostaining in nucleus was classified with a score $>0$ and low with a score 0 . Examination of immunostaining was performed by two independent observers (Z.W. and R.H.) with no knowledge of patient outcome. All discordant scores were reviewed until final agreement was obtained.

\section{Protein extraction}

Protein extraction was performed as described previously [20]. Cells were lysed in ice-cold NP-40 lysis buffer [1\% NP-40, 10\% glycerol, $20 \mathrm{mM}$ Tris- $\mathrm{HCl}, \mathrm{pH} 7.5,137 \mathrm{mM}$ $\mathrm{NaCl}, 100 \mathrm{mM}$ sodium vanadate, $1 \mathrm{mM}$ phenylmethylsulfonyl fluoride (PMSF) and $0.02 \mathrm{mg} / \mathrm{ml}$ each of aprotinin, leupeptin, pepstatin, and $10 \mu \mathrm{l} / \mathrm{ml}$ phosphatase inhibitor cocktail I (Sigma-Aldrich, St. Louis, MO)]. Lysates were sonicated and clarified by centrifugation. Protein quantitation was done by Bradford analysis. Twenty-five $\mu \mathrm{g}$ protein/lane was resolved by $12 \%$ SDS polyacrylamide gel electrophoresis (PAGE) and then Transferred to PVDF membranes.

\section{Western blot analysis}

The PVDF membranes with protein extract from cell lines or $1 \mu \mathrm{g}$ CDC25A, CDC25B and CDC25C antigens (Upstate, NY, USA) were blocked with $5 \%$ nonfat dry milk in tris-buffered saline-Tween (TBST) and subsequently hybridized with antibodies against CDC25s [CDC25A, 1:200; CDC25B, 1:200, (Santa Cruz Biotechnology, CA, USA); CDC25C, 1:200 and phospho-CDC25C (Ser 216), 1:500] overnight at $4^{\circ} \mathrm{C}$, respectively. Membranes were then washed in TBST for 3 times, with 10 mins each, and further hybridized with corresponding anti-mouse or anti-rabbit IgG conjugated with Horseradish Peroxidase (HRP Labelled, 1:5000, $0.2 \mu \mathrm{g} \mathrm{IgG}_{1} / \mathrm{ml}$ dilution) for 1 hour at room temperature. After 3 times rince in TBST for 10 mins each, membranes were finally treated by Western Lightning Enhanced Chemiluminescence (ECL) reagent (Perkin Elmer, MA, USA).

\section{Nuclear and cytoplasmic extraction}

The NE-REP nuclear and cytoplasmic Extraction Reagents (Pierce Biotechnology, Rockford, USA) was used to separate cytoplasm and nuclear proteins. Western blot analysis on each fraction was performed as described above. To confirm the pure separation of nuclear and cytoplasmic fractions, Lamin B, a nuclear protein exclusive recognizing antibody (Pierce Biotechnology), and Tubulin, a cytoplasmic protein exclusive recognizing antibody (Oncogene, San Diego, USA), were used, respectively.

\section{Protein dephosphorylation with CIAP}

Proteins extracted from CAL-39 were dephosphorylated by Calf intestinal alkaline phosphatase (CIAP) (Promega, Madison, WI, USA). Proteins were treated by CIAP in a $50 \mu \mathrm{l}$ reaction volume (protein $5 \mu \mathrm{g}$, CIAP 20 units, $10 \times$ buffer $5 \mu \mathrm{l}$ and $\mathrm{H}_{2} \mathrm{O} 42.5 \mu \mathrm{l}$ ). Following treatment for 30 min at $37^{\circ} \mathrm{C}$, the reaction mixture was added an extra 20 units of CIAP and left for an additional $30 \mathrm{~min}$ at $37^{\circ} \mathrm{C}$. Untreated and CIAP treated proteins were separated by SDS-PAGE and hybridized with $\mathrm{CDC} 25 \mathrm{C}$, phosphoCDC25C (Ser 216), CDC25A and CDC25B antibodies, respectively.

\section{Antigen phosphorylation with CHK1}

One $\mu \mathrm{g}$ antigen $\mathrm{CDC} 25 \mathrm{~B}$ was phosphorylated by $\mathrm{CHK} 1$ (Millipore, Billerica, MA, USA) in a $50 \mu$ reaction volume (antigen CDC25B $1 \mu \mathrm{g}$, CHK1 $20 \mathrm{ng} / \mu \mathrm{l}$, ATP $200 \mu \mathrm{M} / \mu \mathrm{l}$, $10 \times$ kinase buffer $5 \mu \mathrm{l}$ and $\mathrm{H}_{2} \mathrm{O} 39 \mu \mathrm{l}$ ) for $30 \mathrm{~min}$ at room temperature. Untreated and CHK1 treated antigens were separated by SDS-PAGE, blotted onto PVDF membranes and hybridized with $\mathrm{CDC} 25 \mathrm{~B}$ and phospho-CDC25C (Ser 216) antibodies, respectively.

\section{Statistical analyses}

Pearson's chi-square $\left(\mathrm{x}^{2}\right)$ test was performed in order to evaluate associations between CDC25 protein expression and clinicopathologic variables. Kaplan and Meier estimate and the log-rank test were used to evaluate and compare survival data. Disease-specific survival was calculated from the date of diagnosis to vulvar cancer related death. A Cox proportional hazards regression model was used for both univariate and multivariate evaluation of survival rates. In the multivariate analysis, a backward stepwise regression was performed with a $p=$ 0.05 as the inclusion criterion for variables in the univariate analysis. All calculations were processed using SPSS 15.0 statistical software package (SPSS, Chicago, IL) and statistical significance was considered as $p \leq 0.05$. 


\section{Results}

\section{Specificity of antibodies}

The specifity of CDC25 antibodies were tested by Western blot analysis using the antigenes CDC25A, CDC25B and $\mathrm{CDC} 25 \mathrm{C}$. However, since anti-CDC25B used for immunohistochemistry was not recommended by the supplier to use for Western blot analysis, an alternative anti-CDC25B was utilized for Western blot analysis. Our results showed that anti-CDC25A detected CDC25A, but not CDC25B or CDC25C. Anti-CDC25B (only for Western blot analysis) identified CDC25B, but not CDC25A or CDC25C. Anti-CDC25C and anti-phospho-CDC25C (Ser 216) immunoblotted with CDC25C, but not with CDC25A or CDC25B (Figure 1a). These results indicate that anti-CDC25A, anti-CDC25B, anti-CDC25C and anti-phospho-CDC25C (Ser 216) specifically detect their own respective antigen without any crossreaction with other CDC25s isoform.

Anti-CDC25C, as well as anti-CDC25B, detected proteins treated or un-treated with CIAP (Figure 1b), which indicate that anti-CDC25C and anti-CDC25B recognize their respective protein in phosphorylated as well as dephosphorylated form. However, proteins treated with CIAP failed to be identified by anti-CDC25A or antiphospho-CDC25C (Ser 216) (Figure 1b), which indicate that both CDC25A and phospho-CDC25C (Ser 216) antibody exclusively detect phosphorylated CDC25A and CDC25C, respectively.

Anti-CDC25B detected CDC25B antigen treated or untreated with CHK1, but neither of them was identified by anti-phospho-CDC25C (Ser 216) (Figure 1c). Furthermore, no coexpression of $\mathrm{CDC} 25 \mathrm{~B}$ and phosphoCDC25C (Ser216) was seen in serial sections of vulvar carcinomas (Figure 1d-e). These results indicate that the phospho-CDC25C (Ser 216) antibody does not crossreact with phosphorylated CDC25B.

\section{CDC25s protein expression}

In normal vulvar squamous epithelium, nuclear membrane staining for CDC25A was identified in basal, parabasal, middle and top layers (10/10 cases with score 9), whereas nuclear staining for CDC25B was seen in basal, parabasal and middle layers (10/10 cases with score 6) (Figure 2a-b). Cytoplasmic staining for CDC25C was observed in basal, parabasal and middle layers ( $3 / 10$ cases with score 3 and 7/10 cases with score 6), whereas phospho-CDC25C (Ser 216) was limited to the basal layer (7/ 10 cases negative and 3/10 cases with score 3 ) (Figure 2cd). None of the normal cases showed nuclear staining for CDC25C and phospho-CDC25C (Ser 216).

The immunostaining results in vulvar carcinomas are summarized in Table 1. High CDC25A and CDC25B immunostaining (score $>6$ ) in the nucleus was observed in $152 / 300(51 \%)$ and $47 / 300(16 \%)$ cases, respectively
(Figure 2e-f). CDC25A expression was observed in the nuclear membrane. High CDC25C expression (score > 3) in the cytoplasm was seen in 188/300 (63\%) cases (Figure $2 \mathrm{~g})$. CDC25C immunostaining in the nucleus was not observed in any cases. High phospho-CDC25C (Ser216) expression in cytoplasm (score $>3$ ) and nucleus (score > 0 ) were detected in 151/300 (50\%) and 211/300 (70\%) cases, respectively. Taking both the cytoplasmic and nuclear immunostaining into account, we found that high phospho-CDC25C (Ser216) immunostaining (score > 3) appeared in 232/300 (77\%) cases (Figure 2h).

In the vulvar carcinoma cell lines SW-954 and CAL-39, immunohistochemistry identified CDC25A (score $=3$ ), CDC25B (score $=9$ ), CDC25C (score $=3$ ) and phosphoCDC25C (Ser216) (score $=3$ ) in the nucleus, whereas, CDC25B (score $=3$ ), CDC25C (SW-954, score $=6$ and CAL-39, score $=9$ ), and phospho-CDC25C (Ser216) $($ score $=9)$ were observed in the cytoplasm (Figure 3a-d). Similar results were detected using Western blot analysis (Figure 3e). CDC25A was seen only in the nuclear fraction. CDC25B was weakly expressed in the cytoplasmic and strongly in the nuclear fraction. In contrast, CDC25C and phospho-CDC25C (Ser 216) were strongly expressed in the cytoplasmic fraction, but weakly in the nuclear fraction.

\section{Immunostaining results of CDC25s in relation to clinicopathological parameters}

High expression of CDC25A in the nucleus was significantly correlated to low expression of $14-3-3 \sigma$ protein in cytoplasm, nucleus and cytoplasm/nucleus ( $p=0.03, p=$ 0.01 and $p=0.01$, respectively) and to low expression of phospho-CDC25C (Ser216) in nucleus and cytoplasm/ nucleus $(p=0.002$ and $p=0.04$, respectively). High expression of CDC25B in the nucleus was significantly correlated with poor differentiation $(p=0.004)$, vessel infiltration $(p=0.03)$, high expression of phosphoCDC25C (Ser216) in cytoplasm and cytoplasm/nucleus ( $p=0.01$, and $p=0.04$ respectively) and HPV infected cases $(p=0.03)$. The levels of CDC25C and phosphoCDC25C (Ser216) immunostaining in relation to clinicopathological parameters are shown in Table 2. High expression of $\mathrm{CDC} 25 \mathrm{C}$ in cytoplasm was significantly correlated with high FIGO substage $(p=0.004)$, presence of lymph node metastases $(p=0.04)$, large tumor diameter $(p=0.03)$, poor differentiation $(p=0.03)$, high expression of $14-3-3 \sigma$ protein in cytoplasm and cytoplasm/ nucleus (both $p=0.001$ ) and high expression of phosphoCDC25C (Ser216) in cytoplasm, nucleus and cytoplasm/ nucleus (all $p<0.001$ ). High expression of phosphoCDC25C (Ser216) in cytoplasm, nucleus and cytoplasm/ nucleus were significantly correlated with high FIGO substage ( $p=0.05, p=0.01$ and $p=0.005$, respectively), large tumor diameter $(p<0.001, p=0.009$ and $p<0.001$, 


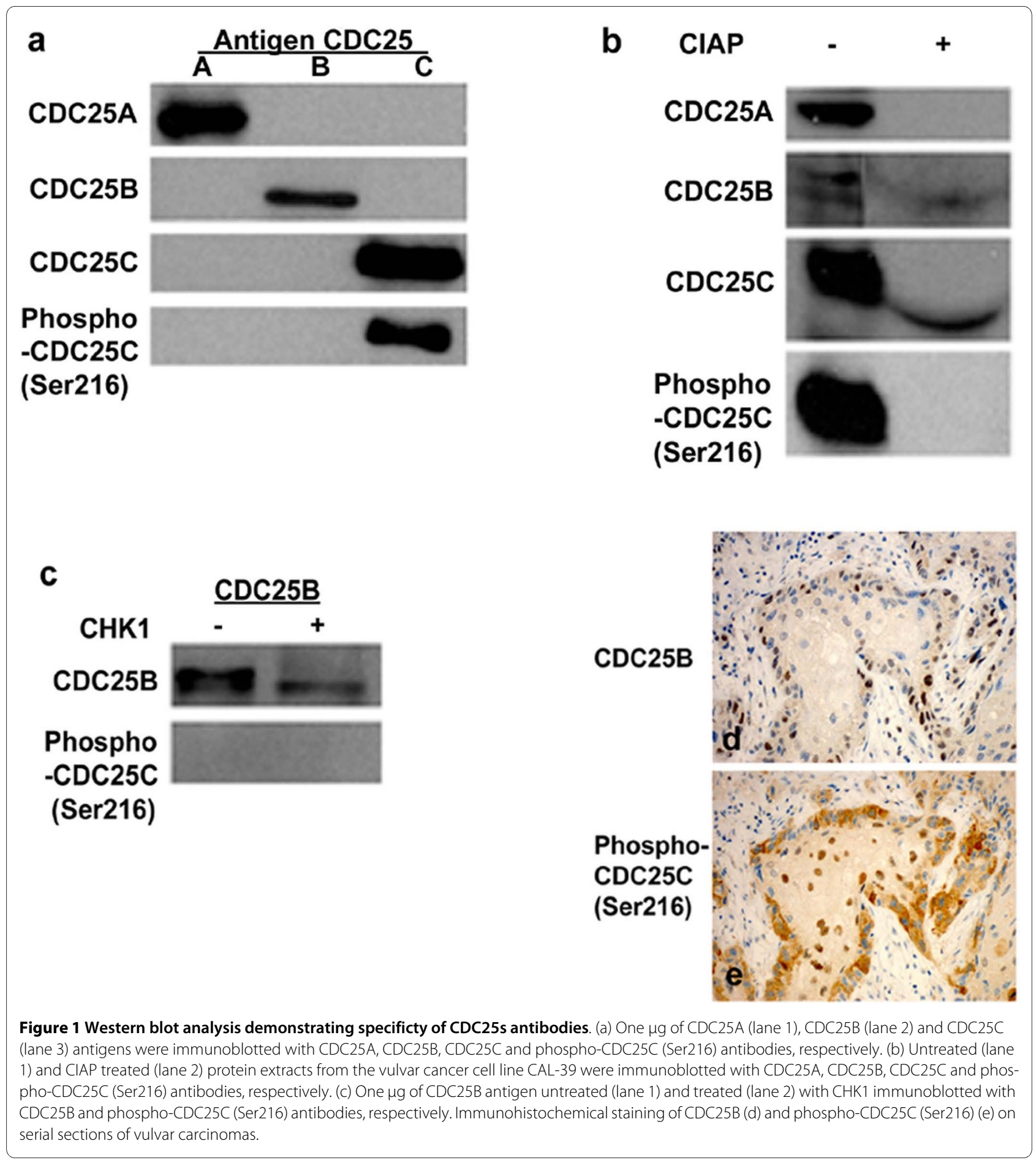

respectively), deep invasion ( $p=0.01, p=0.01$ and $p<$ 0.001 , respectively), high $14-3-3 \sigma$ protein levels in cytoplasm ( $p=0.007, p<0.001$ and $p<0.001$, respectively) and high $14-3-3 \sigma$ protein expression in cytoplasm/ nucleus ( $p=0.005, p<0.001$ and $p<0.001$, respectively). Factors showing significant correlation with high expression of phospho-CDC25C (Ser216) in cytoplasm and cytoplasm/nucleus were poor differentiation $(p<0.001$ and $p=0.01$, respectively). High nuclear expression of 143-3 $\sigma$ was significantly correlated with high expression of phospho-CDC25C (Ser216) in nucleus $(p<0.001)$ and cytoplasm/nucleus $(p=0.002)$. Cases negative for HPV was significantly correlation with high expression of phospho-CDC25C (Ser216) in nucleus $(p<0.001)$.

In the univariate analysis only high expression of phospho-CDC25C (Ser216) in cytoplasm/nucleus was associ- 


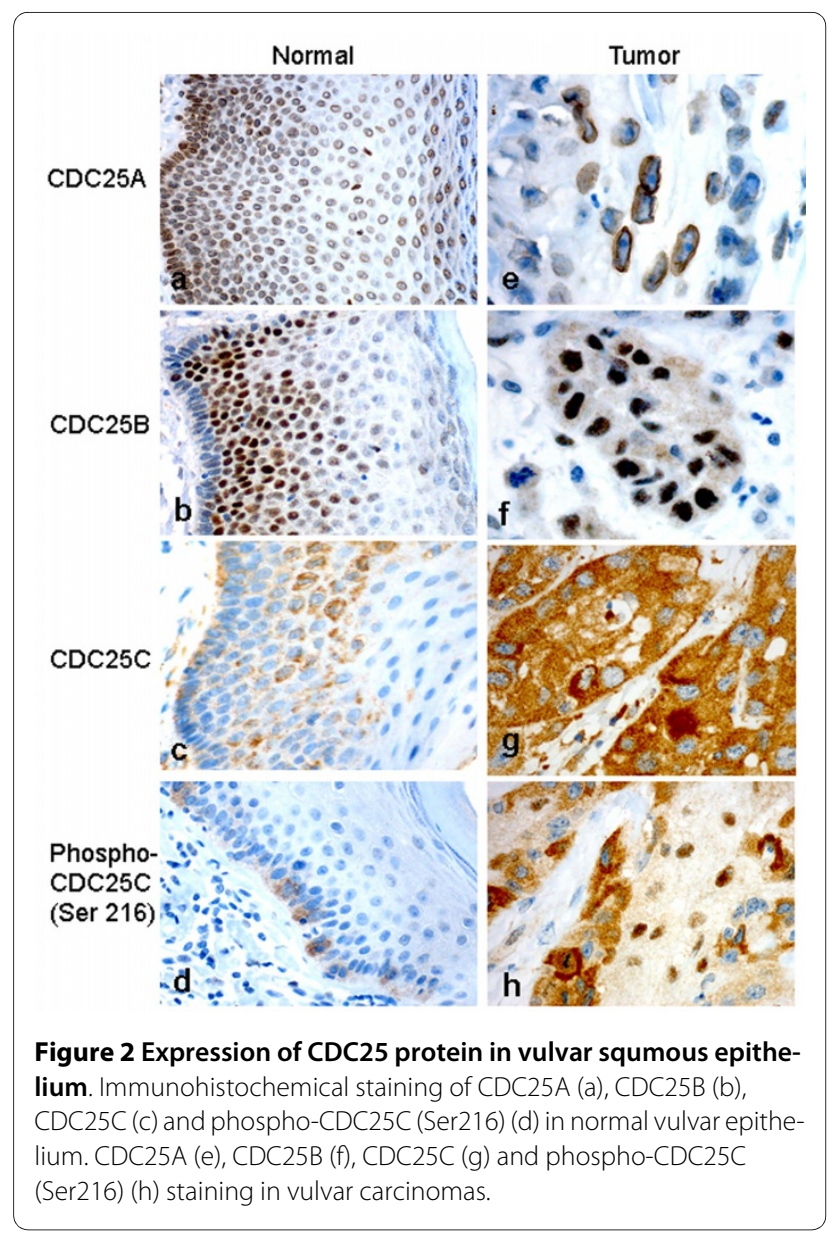

ated with poor disease-specific survival $(p=0.04)$ (Figure $4)$. However, in multivariate analysis, when phosphoCDC25C (Ser216) cytoplasm/nucleus expression was added to the variables lymph node metastases, tumor diameter, infiltration of vessel, age and depth of invasion, only lymph node metastases, tumor diameter, vessel infil- tration and age retained independent prognostic significance (Table 3).

\section{Discussion}

Overexpression of CDC25A has been reported in breast [12], esophageal [14], hepatocellular [21], colorectal [9] and ovarian carcinomas [13]. Therefore, abnormal expression of CDC25A was implied as one of the potential oncogenic factors leading to dysregulation of cell cycle control and consequently oncogenic transformation. However, in our study overexpression of CDC25A was found not only in $51 \%$ of the vulvar carcinomas, but also in normal vulvar squamous epithelium, indicating that high expression of CDC25A is not important in the pathogenesis of vulvar carcinomas. In agreement with studies on thyroid [22] and colorectal carcinomas [9], we found that disease-free survival was not significantly associated with high expression of CDC25A, although such an association was reported in breast [12], oesophageal [14] and hepatocellular carcinomas [21]. These results indicate that the role of $\mathrm{CDC} 25 \mathrm{~A}$ is cancer specific.

In our study, $16 \%$ of the vulvar carcinomas have higher expression of CDC25B than normal vulvar squamous epithelium. Previously, a wide range of CDC25B overexpression $(20-79 \%)$ has been reported in many other cancer types [5]. This discrepancy may be due to the various tumor types studied. Overexpression of CDC25B in 16\% of our cases suggests that this protein may contribute to tumorigenesis in a minority of vulvar carcinomas. We failed to find an association with high CDC25B expression and disease-free survival, which was also reported in studies on prostate [23], gastric [24], hepatocellular [21], breast [12] and endometrial carcinomas. In contrast, a positive correlation was found in colorectal [9] and ovarian carcinomas [13]. These results suggest that contro-

Table 1: Immunostaining results for CDC25s

\begin{tabular}{|c|c|c|c|c|c|c|}
\hline \multirow[t]{3}{*}{ Score } & \multirow{2}{*}{$\begin{array}{l}\text { CDC25A } \\
\text { Nucleus }\end{array}$} & \multirow{2}{*}{$\begin{array}{l}\text { CDC25B } \\
\text { Nucleus }\end{array}$} & \multirow{2}{*}{$\begin{array}{c}\text { CDC25C } \\
\text { Cytoplasm }\end{array}$} & \multicolumn{3}{|c|}{ Phospho-CDC25C (Ser216) } \\
\hline & & & & Cytoplasm & Nucleus & $\begin{array}{l}\text { Cytoplasm } \\
\text { and nucleus }\end{array}$ \\
\hline & n (\%) & n (\%) & n (\%) & n (\%) & n (\%) & n (\%) \\
\hline 0 & $3(1.0)$ & $2(0.7)$ & $13(4.3)$ & $54(18.0)$ & $89(29.7)$ & $18(6.0)$ \\
\hline 2 & $0(0)$ & $6(2.0)$ & $5(1.7)$ & $15(5.0)$ & $7(2.3)$ & $8(2.7)$ \\
\hline 3 & $35(11.7)$ & $36(12.0)$ & $94(31.3)$ & $80(26.7)$ & $46(15.3)$ & $42(14.0)$ \\
\hline 4 & $1(0.3)$ & $6(2.0)$ & $17(5.7)$ & $17(5.7)$ & $10(3.3)$ & $13(4.3)$ \\
\hline 6 & $109(36.3)$ & $203(67.7)$ & $135(45.0)$ & $125(41.7)$ & $112(37.3)$ & $143(47.7)$ \\
\hline 9 & $152(50.7)$ & $47(15.7)$ & $36(12.0)$ & $9(3.0)$ & $36(12.0)$ & $76(25.3)$ \\
\hline Total & $300(100.0)$ & $300(100.0)$ & $300(100.0)$ & $300(100.0)$ & $300(100.0)$ & $300(100.0)$ \\
\hline
\end{tabular}




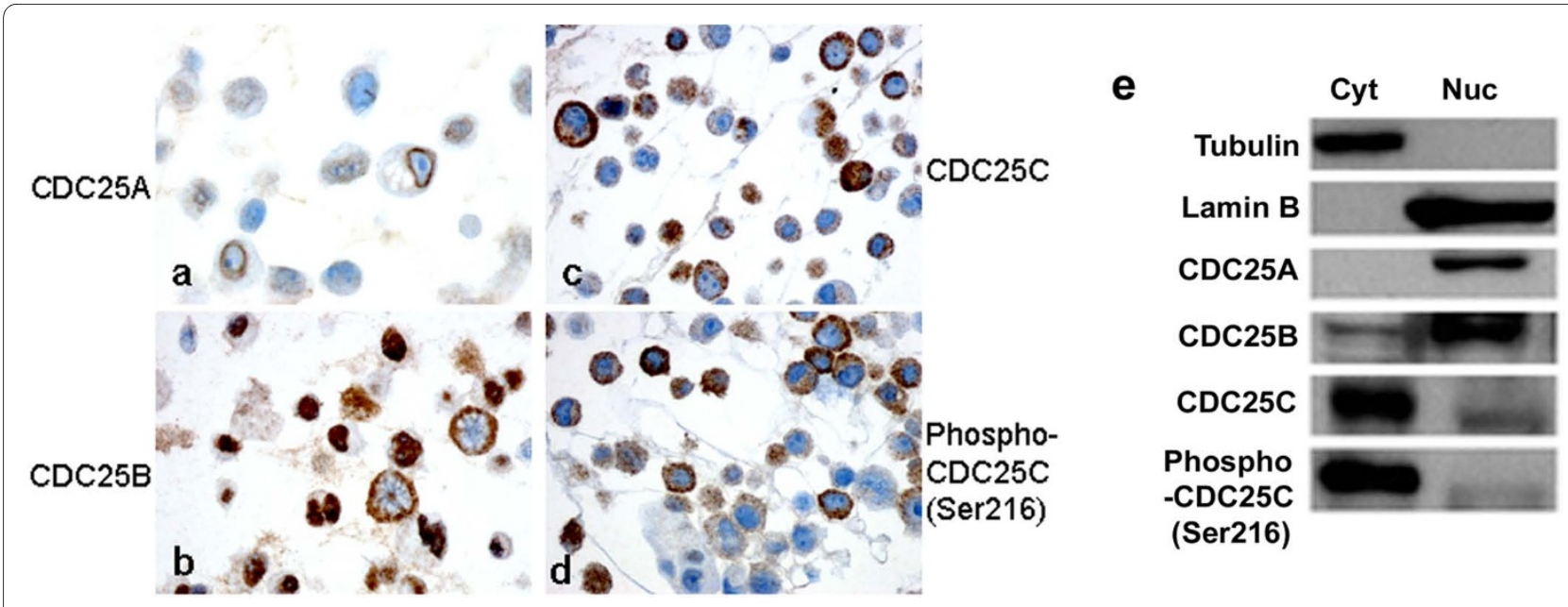

Figure 3 Expression of CDC25 protein in vulvar cancer cell line. Immunohistochemical staining of CDC25A (a), CDC25B (b), CDC25C (c) and phospho-CDC25C (Ser216) (d) in CAL-39 cell line. Western blot analysis of CDC25s cellular localization in CAL-39 cells (e). Cell fractions of cytoplasm (lane 1) and nucleus (lane 2) were immunoblotted with Tubulin and Lamin B antibodies to verify the purity of subcellular fraction extraction, then with CDC25A, CDC25B, CDC25C and phospho-CDC25C (Ser216) antibodies, respectively. Cyt: Cytoplasmic fraction; Nuc: Nuclear fraction.

versy still rests on CDC25B as a universal tumor marker and that its prognostic value on gynaecologic carcinomas seems limited.

In previous studies, overexpression of $\mathrm{CDC} 25 \mathrm{C}$ has been reported in a limited number of carcinomas. Therefore, $\mathrm{CDC} 25 \mathrm{C}$ was regarded as a less oncogenic factor than CDC25A and CDC25B [5]. However, in the present study high expression of CDC25C was observed in $63 \%$ of vulvar carcinomas, a finding in line with studies on prostate [15], colorectal [25] and endometrium carcinomas [10]. The fact that overexpression of $\mathrm{CDC} 25 \mathrm{C}$ was associated with advanced FIGO stage, presence of lymph node metastases, large tumor diameter and poor differentiation indicate that high level of $\mathrm{CDC} 25 \mathrm{C}$ is an event occuring late in the tumor development. Our study failed to show a positive correlation between overexpression of CDC25C and disease-specific survival, which was similar to the results reported in esophageal [26], pancreatic [27], gastric [24] and ovarian carcinomas [13].

Compared to the low level of cytoplasmic phosphoCDC25C (Ser 216) protein expression in basal layers of normal vulvar squamous epithelium, high phosphoCDC25C (Ser 216) protein expression was found in the cytoplasm of $50 \%$ and in the nucleus of $70 \%$ of vulvar carcinomas. High expression of cytoplamic phosphoCDC25C (Ser 216) was correlated with high level of cytoplasmic 14-3-3 $\sigma$. This strengthens the theory that phospho-CDC25C (Ser 216) protein sequestrated in the cytoplasm due to binding of 14-3-3 lose its access to nuclear CDK1/cyclin B complex, thus inhibiting mitotic entry [5]. Unexpectly, high expression of nuclear phospho-CDC25C (Ser 216) was correlated with high expression of nuclear 14-3-3 $\sigma$ protein. One hypothesis is that phospho-CDC25C (Ser 216) in the nucleus is unable to bind 14-3-3б [28]. Phospho-CDC25C (Ser 216) will then still stay in the nucleus and activate CDK1/cyclin B complex, thus triggering G2/M transition. Therefore, high nuclear expressions of phospho-CDC25C (Ser 216) in the majority of cases indicate that phospho-CDC25C (Ser 216) may be important in the carcinogenesis of vulvar carcinomas and would be a potential target for cancer therapy.

According to the analyses between phospho-CDC25C (Ser 216) and clinical parameters, high expression of phospho-CDC25C (Ser 216) in cytoplasm/nucleus was significantly correlated with advanced FIGO stage, large tumor diameter and deep invasion as well as poor disease-specific survival. However, such an association between phospho-CDC25C (Ser 216) expression in cytoplasm/nucleus and disease-specific survival was annulled in multivariate analysis. The phospho-CDC25C (Ser 216) has to our knowledge not been previously investigated in any human cancer. Therefore, further studies are needed to clarify the role of phospho-CDC25C (Ser 216) as a prognostic marker.

In the present study, overexpression of CDC25A, $\mathrm{CDC} 25 \mathrm{~B}$ and CDC25C isoforms was not significant associated with each other, suggesting that overexpression of multiple isoforms in vulvar carcinomas occur through independent pathways [5]. However, high phosphoCDC25C (Ser 216) expression was correlated with low expression of CDC25A and high expression of CDC25B, suggesting that the three can collaborate in the tumorigenesis of a subset of vulvar carcinomas. Firstly, low expression of CDC25A in $49 \%$ of vulvar, compared to its high expression in normal tissues, might account for DNA damage-induced G2 arrest which is accompanied by proteasome-dependent destruction of CDC25A $[7,29]$. 
Table 2: CDC25C and phospho-CDC25C (Ser216) immunostaining in relation to clinicopathological variables

\begin{tabular}{|c|c|c|c|c|c|c|c|c|c|c|}
\hline \multirow[t]{3}{*}{ Variables } & \multirow{3}{*}{$\begin{array}{c}\text { Total } \\
\text { n }\end{array}$} & \multirow{2}{*}{\multicolumn{3}{|c|}{$\begin{array}{c}\text { CDC25C } \\
\text { Cytoplasm }\end{array}$}} & \multicolumn{6}{|c|}{ Phospho-CDC25C (Ser216) } \\
\hline & & & & & \multicolumn{3}{|c|}{ Cytoplasm } & \multicolumn{3}{|c|}{ Nucleus } \\
\hline & & Low & High (\%) & $p^{1}$ & Low & High (\%) & $p^{1}$ & Low & High (\%) & $p^{1}$ \\
\hline Age & & & & 0.91 & & & 0.91 & & & 0.45 \\
\hline $25-69$ & 119 & 46 & $73(61)$ & & 61 & $58(49)$ & & 40 & $79(66)$ & \\
\hline $70-84$ & 147 & 53 & $94(64)$ & & 71 & $76(52)$ & & 39 & $108(74)$ & \\
\hline $85+$ & 34 & 13 & $21(62)$ & & 17 & $17(50)$ & & 10 & $24(71)$ & \\
\hline FIGO & & & & 0.004 & & & 0.05 & & & 0.01 \\
\hline la & 11 & 5 & $6(55)$ & & 7 & $4(36)$ & & 6 & $5(46)$ & \\
\hline $\mathrm{lb}$ & 35 & 23 & $12(34)$ & & 24 & $11(31)$ & & 17 & $18(51)$ & \\
\hline ॥ & 110 & 38 & $72(66)$ & & 55 & $55(50)$ & & 25 & $85(77)$ & \\
\hline III & 121 & 38 & $83(69)$ & & 55 & $66(55)$ & & 32 & $89(74)$ & \\
\hline IV & 19 & 6 & $13(68)$ & & 6 & $13(68)$ & & 6 & $13(68)$ & \\
\hline Not available & 4 & & & & & & & & & \\
\hline Lymph node metastases & & & & 0.04 & & & 0.14 & & & 0.54 \\
\hline None & 136 & 58 & $78(57)$ & & 78 & $58(43)$ & & 40 & $96(71)$ & \\
\hline Unilateral & 76 & 21 & $55(72)$ & & 33 & $43(57)$ & & 19 & $57(75)$ & \\
\hline Bilateral & 34 & 9 & $25(74)$ & & 16 & $18(53)$ & & 7 & $27(79)$ & \\
\hline Not available & 54 & & & & & & & & & \\
\hline Tumour diameter $(\mathrm{cm})$ & & & & 0.03 & & & $<0.001$ & & & 0.009 \\
\hline $0.3-2.5$ & 90 & 41 & $49(54)$ & & 60 & $30(33)$ & & 35 & $55(61)$ & \\
\hline $2.6-4.0$ & 94 & 34 & $60(64)$ & & 46 & $48(51)$ & & 21 & $73(78)$ & \\
\hline $4.1-20.0$ & 100 & 27 & $73(73)$ & & 35 & $65(65)$ & & 21 & $79(79)$ & \\
\hline Not available & 16 & & & & & & & & & \\
\hline Tumor differentiation & & & & 0.03 & & & $<0.001$ & & & 0.30 \\
\hline Well & 74 & 37 & $37(50)$ & & 51 & $23(31)$ & & 18 & $56(76)$ & \\
\hline Moderate & 154 & 53 & $101(66)$ & & 74 & $80(52)$ & & 45 & $109(71)$ & \\
\hline Poor & 72 & 22 & $50(69)$ & & 24 & $48(67)$ & & 26 & $46(64)$ & \\
\hline Depth of invasion (mm) & & & & 0.08 & & & 0.01 & & & 0.01 \\
\hline $0.0-4.0$ & 79 & 34 & $45(57)$ & & 50 & $29(37)$ & & 32 & $47(60)$ & \\
\hline $4.1-8.0$ & 98 & 27 & $71(72)$ & & 48 & $50(51)$ & & 21 & $77(79)$ & \\
\hline $8.1-40.0$ & 112 & 44 & $68(61)$ & & 46 & $66(59)$ & & 27 & $85(76)$ & \\
\hline Not available & 11 & & & & & & & & & \\
\hline Infiltration of vessel & & & & 0.32 & & & 0.26 & & & 0.54 \\
\hline No & 232 & 91 & $141(61)$ & & 119 & $113(49)$ & & 71 & $161(69)$ & \\
\hline Yes & 65 & 21 & $44(68)$ & & 28 & $37(57)$ & & 17 & $48(74)$ & \\
\hline Not available & 3 & & & & & & & & & \\
\hline $\mathrm{HPV}^{2}$ & & & & 0.29 & & & 0.09 & & & $<0.001$ \\
\hline Low (-) & 167 & 57 & $110(66)$ & & 99 & $68(41)$ & & 32 & $135(81)$ & \\
\hline High (+) & 43 & 19 & $24(56)$ & & 19 & $24(56)$ & & 21 & $22(51)$ & \\
\hline Not available & 90 & & & & & & & & & \\
\hline
\end{tabular}


Table 2: CDC25C and phospho-CDC25C (Ser216) immunostaining in relation to clinicopathological variables (Continued)

\begin{tabular}{|c|c|c|c|c|c|c|c|c|c|c|}
\hline 14-3-30 cytoplasm² & & & & 0.001 & & & 0.007 & & & $<0.001$ \\
\hline $\operatorname{Low}(<6)$ & 83 & 43 & $40(48)$ & & 52 & $31(37)$ & & 39 & $44(53)$ & \\
\hline High ( $\geq 6)$ & 217 & 69 & $148(68)$ & & 97 & $120(55)$ & & 50 & $167(77)$ & \\
\hline $14-3-3 \sigma$ nucleus $^{2}$ & & & & 0.40 & & & 0.48 & & & $<0.001$ \\
\hline $\operatorname{Low}(<6)$ & 123 & 42 & $81(66)$ & & 58 & $65(53)$ & & 51 & $72(59)$ & \\
\hline High ( $\geq 6)$ & 177 & 70 & $107(61)$ & & 91 & $86(49)$ & & 38 & $139(79)$ & \\
\hline $\begin{array}{l}\text { 14-3-30 cytoplasm/ } \\
\text { nucleus }^{2}\end{array}$ & & & & 0.001 & & & 0.005 & & & $<0.001$ \\
\hline $\operatorname{Low}(<6)$ & 75 & 40 & $35(47)$ & & 48 & $27(36)$ & & 36 & $39(52)$ & \\
\hline $\operatorname{High}(\geq 6)$ & 225 & 72 & $153(68)$ & & 101 & $124(55)$ & & 53 & $172(76)$ & \\
\hline CDC25A & & & & 0.48 & & & 0.49 & & & 0.002 \\
\hline Low & 148 & 52 & $96(65)$ & & 77 & $71(48)$ & & 31 & $117(79)$ & \\
\hline High & 152 & 60 & $92(61)$ & & 72 & $80(53)$ & & 58 & $94(62)$ & \\
\hline CDC25B & & & & 0.87 & & & 0.01 & & & 0.13 \\
\hline Low & 253 & 95 & $158(63)$ & & 134 & $119(47)$ & & 74 & $179(71)$ & \\
\hline High & 47 & 17 & $30(64)$ & & 15 & $32(68)$ & & 15 & $32(68)$ & \\
\hline CDC25C & & & & - & & & $<0.001$ & & & $<0.001$ \\
\hline Low & 112 & - & - - & & 76 & $36(32)$ & & 47 & $65(58)$ & \\
\hline High & 188 & - & - - & & 73 & $115(61)$ & & 42 & $146(78)$ & \\
\hline
\end{tabular}

1 Pearson chi-square

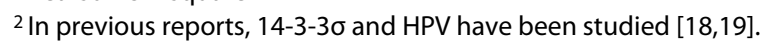

Secondly, CDC25C phosphorylated at Ser 216 in response to DNA damage [7] stay in the nucleus, instead of being sequestrated in cytoplasm, and might still be able to phosphorylate its substrate CDK1/cyclin B complex, resulting in an un-thorough $\mathrm{G} 2$ arrest. This un-thorough G2 arrest might be enhanced by overexpression of CDC25B which, other than A and C, was essential for mitotic entry as cells recover from a DNA-induced checkpoint arrest [7,30-33].

Our result showed that infection of HPV correlated with high expression of CDC25B and nuclear phosphoCDC25C (Ser216). This result is in agreement with previous studies of CDC25B, where CDC25B mRNA was highly elevated in fibroblasts after being transformed by SV-40 or by E6 or E7 papilloma virus transforming proteins [5,34]. Little is known about CDC25C post-transcriptional changes due to virally induced cellular transformation. However, we found an increased CDC25C protein expression in presence of HPV infection. In our study we could not demonstrate an association between CDC25A protein and HPV infection, although an elevated mRNA level and enzyme activity of CDC25A was found in quiescent human fibroblasts infected with the EIA adenovirus protein [35]. These findings suggest that CDC25s promoters may be specifically targeted by viruses during the cell transformation process [5] and that CDC25B and CDC25C may be subjected to HPV regulation.
Human CDC25 proteins consist of two domains: the Nterminal regulatory domain where the three isoforms share $20-25 \%$ identity and the C-terminal catalytic domain sharing approximately $60 \%$ identity [7]. Due to the similarity in the structure, antibodies to CDC25A, CDC25B and CDC25C have a high potential to not only identify their specific antigen, but also the other two isoforms. We found that the four CDC25 antibodies used in the present study detected their own respective antigen without any cross-reaction with the other CDC25s, strengthening the trustiness of our results. In contrast, previous papers have used CDC25s antibodies for immunohistochemistry without knowing the specificity of their antibodies [8,10,14,22,23,27,36,37], which may partly explain the conflicting results regarding cellular localization of CDC25A, CDC25B and CDC25C [6].

Interestingly, a different immunostaining pattern was seen between the two CDC25C antibodies in vulvar carcinomas. By using anti-CDC25C, which recognized both phosphorylated and dephosphorylated forms, immunostaining was detected only in cytoplasm, whereas antiphospho-CDC25C (Ser 216), which only recognizes phosphorylated form, immunostained both in cytoplasm and nucleus. Previously, it has been reported that six amino acids are homologous in the phospho-CDC25C (Ser 216) and phospho-CDC25B (Ser323) domain [38]. Therefore, we were led to believe that the nuclear immunostaining observed by using anti-phospho-CDC25C 
Table 3: Relative risk (RR) of dying from vulvar cancer

\begin{tabular}{|c|c|c|c|c|c|c|}
\hline \multirow[t]{2}{*}{ Variables } & \multicolumn{3}{|c|}{ Univariate analysis } & \multicolumn{3}{|c|}{ Multivariate analysis } \\
\hline & $\mathbf{R R}$ & $95 \% \mathrm{Cla}^{\mathrm{a}}$ & $p$ & $\mathbf{R R}$ & $95 \% \mathrm{Cla}^{\mathrm{a}}$ & $\boldsymbol{p}$ \\
\hline $\begin{array}{l}\text { Lymph node } \\
\text { metastases }\end{array}$ & 2.49 & $1.92-3.23$ & $<0.001$ & 2.18 & $1.64-2.90$ & $<0.001$ \\
\hline $\begin{array}{l}\text { Tumour } \\
\text { diameter }\end{array}$ & 1.78 & $1.40-2.25$ & $<0.001$ & 1.47 & $1.10-1.95$ & 0.009 \\
\hline $\begin{array}{l}\text { Infiltration of } \\
\text { vessel }\end{array}$ & 2.42 & $1.64-3.57$ & $<0.001$ & 1.76 & $1.10-2.82$ & 0.02 \\
\hline Age & 1.60 & $1.22-2.10$ & 0.001 & 1.46 & $1.03-2.08$ & 0.03 \\
\hline $\begin{array}{l}\text { Phospho- } \\
\text { CDC25C } \\
(\text { Ser216) }\end{array}$ & 1.64 & $1.02-2.64$ & 0.04 & - & - & - \\
\hline
\end{tabular}

(Ser 216) was false due to cross-reaction with phosphoCDC25B (Ser323). However, this was not the case, since we excluded the possibility that anti-phospho-CDC25C (Ser 216) cross-react with phosphorylated CDC25B by the kinase test and immunostaining. On serial sections, no coexpression was observed between phosphoCDC25C (Ser 216) and CDC25B, both phosphorylated and dephosphorylated forms. Therefore, we speculated that in vulvar carcinomas the epitope in the phosphoCDC25C (Ser 216) domain supposed to be recognized by anti-CDC25C (phosphorylated and dephosphorylated) may be masked for some unknown reasons resulting in lost nuclear staining.

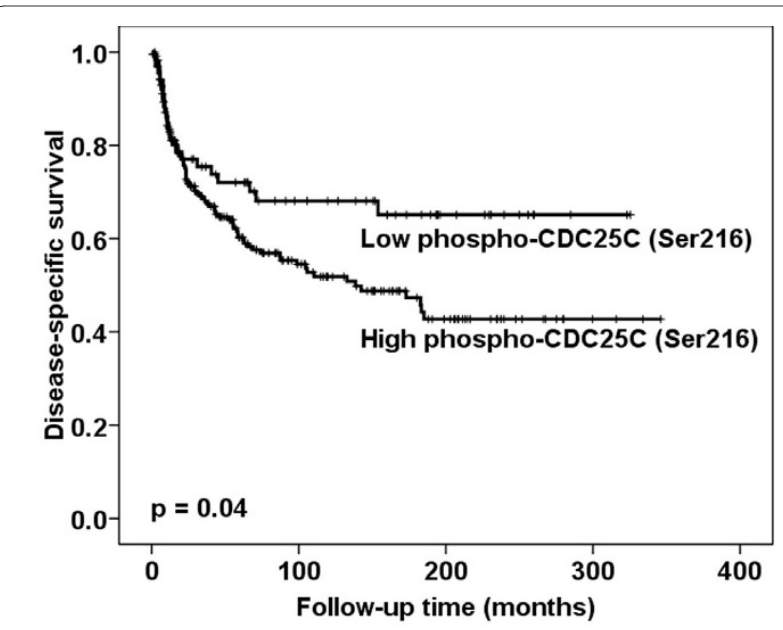

Figure 4 Survival curves using the Kaplan-Meier method. KaplanMeier curve of disease-specific survival in relation to phospho-CDC25C (Ser216) protein expression levels in cytoplasm/nucleus $(p=0.04)$ for the entire series $(n=300)$.

\section{Conclusions}

Our results suggest that $\mathrm{CDC} 25 \mathrm{C}$ and phospho-CDC25C (Ser216) play a crucial role and CDC25B a minor role in the development and/or progression of vulvar carcinomas. CDC25B, CDC25C and phospho-CDC25C (Ser216) expression were associated with malignant features and aggressive cancer phenotypes. However, the CDC25s isoforms were not independently correlated to prognosis.

\section{Competing interests}

The authors declare that they have no competing interests.

\section{Authors' contributions}

ZW participated in the design of the study, carried out the immunohistochemistry, immunoblotting, statistical and data analysis and draft the manuscript. CGT collected clinical data, participated in interpretation of data and helped to draft the manuscript. VAF participated in immunoblotting analysis and interpretation and revised the manuscript critically. ZS participated in the design of the study and revised the manuscript critically. JMN performed systematic pathologic review of vulvar carcinomas and revised the manuscript critically. $\mathrm{RH}$ participated in the design of the study, protein, statistical and data analysis and helped to draft the manuscript. All authors read and approved the final manuscript.

\section{Acknowledgements}

We thank Mette Førsund, Anne-Marie Becker, Liv Inger Håseth, Ellen Hellesylt and Anne Katrine Rosnes for excellent technical support. This project was supported by grants from The Norwegian Cancer Society and Health Region South of Norway. Zhihui Wang is a research fellow of The Norwegian Cancer Society.

\section{Author Details}

1Department of Pathology, Oslo University Hospital and University of Oslo, Norway, ${ }^{2}$ Department of Oncology, The First Affiliated Hospital of Zhengzhou University, Medical College of Zhengzhou University, Zhengzhou, China, ${ }^{3}$ Department of Obstetrics and Gynecology, Oslo University Hospital and University of Oslo, Norway and ${ }^{4}$ Department of Pathology, Oslo University Hospital, Norway

Received: 15 December 2009 Accepted: 25 May 2010 Published: 25 May 2010 


\section{References}

1. Coulter J, Gleeson N: Local and regional recurrence of vulval cancer: management dilemmas. Best Pract Res Clin Obstet Gynaecol 2003 17:663-681.

2. Jones RW, Baranyai J, Stables S: Trends in squamous cell carcinoma of the vulva: the influence of vulvar intraepithelial neoplasia. Obstet Gynecol 1997, 90:448-452.

3. Messing MJ, Gallup DG: Carcinoma of the vulva in young women. Obstet Gynecol 1995, 86:51-54.

4. Tyring SK: Vulvar squamous cell carcinoma: guidelines for early diagnosis and treatment. Am J Obstet Gynecol 2003, 189:S17-S23.

5. Boutros R, Lobjois V, Ducommun B: CDC25 phosphatases in cancer cells: key players? Good targets? Nat Rev Cancer 2007, 7:495-507.

6. Kristjansdottir K, Rudolph J: Cdc25 phosphatases and cancer. Chem Biol 2004, 11:1043-1051.

7. Aressy B, Ducommun B: Cell cycle control by the CDC25 phosphatases. Anticancer Agents Med Chem 2008, 8:818-824

8. Sasaki H, Yukiue H, Kobayashi Y, Tanahashi M, Moriyama S, Nakashima Y, Fukai I, Kiriyama M, Yamakawa Y, Fujii Y: Expression of the cdc25B gene as a prognosis marker in non-small cell lung cancer. Cancer Lett 2001, 173:187-192.

9. Takemasa I, Yamamoto $H$, Sekimoto $M$, Ohue $M$, Noura S, Miyake $Y$, Matsumoto T, Aihara T, Tomita N, Tamaki Y, Sakita I, Kikkawa N, Matsuura $\mathrm{N}$, Shiozaki H, Monden M: Overexpression of CDC25B phosphatase as a novel marker of poor prognosis of human colorectal carcinoma. Cancer Res 2000, 60:3043-3050.

10. Tsuda H, Hashiguchi $Y$, Inoue T, Yamamoto K: Alteration of $G 2$ cell cycle regulators occurs during carcinogenesis of the endometrium. Oncology 2003, 65:159-166.

11. Galaktionov K, Lee AK, Eckstein J, Draetta G, Meckler J, Loda M, Beach D: CDC25 phosphatases as potential human oncogenes. Science 1995, 269:1575-1577

12. Cangi MG, Cukor B, Soung P, Signoretti S, Moreira G Jr, Ranashinge M, Cady B, Pagano M, Loda M: Role of the Cdc25A phosphatase in human breast cancer. J Clin Invest 2000, 106:753-761.

13. Broggini M, Buraggi G, Brenna A, Riva L, Codegoni AM, Torri V, Lissoni AA, Mangioni C, D'Incalci M: Cell cycle-related phosphatases CDC25A and B expression correlates with survival in ovarian cancer patients. Anticancer Res 2000, 20:4835-4840.

14. Nishioka K, Doki Y, Shiozaki H, Yamamoto H, Tamura S, Yasuda T, Fujiwara Y, Yano M, Miyata H, Kishi K, Nakagawa H, Shamma A, Monden M: Clinical significance of CDC25A and CDC25B expression in squamous cell carcinomas of the oesophagus. Br J Cancer 2001, 85:412-421.

15. Ozen M, Ittmann M: Increased expression and activity of CDC25C phosphatase and an alternatively spliced variant in prostate cancer. Clin Cancer Res 2005, 11:4701-4706.

16. Shepherd $\mathrm{JH}$ : Cervical and vulva cancer: changes in FIGO definitions of staging. Br J Obstet Gynaecol 1996, 103:405-406.

17. WHO: Pathology and Genetics: Tumours of the Breast and Female Genital Organs. In World Health Organization Classification of Tumors Lyon: IARC Press; 2003:316-321

18. Knopp S, Nesland JM, Trope C, Holm R: p14ARF, a prognostic predictor in HPV-negative vulvar carcinoma. Am J Clin Pathol 2006, 126:266-276.

19. Wang Z, Trope CG, Suo Z, Troen G, Yang G, Nesland JM, Holm R: The clinicopathological and prognostic impact of 14-3-3 sigma expression on vulvar squamous cell carcinomas. BMC Cancer 2008, 8:308.

20. Jorgensen K, Skrede M, Cruciani V, Mikalsen SO, Slipicevic A, Florenes VA Phorbol ester phorbol-12-myristate-13-acetate promotes anchorageindependent growth and survival of melanomas through MEKindependent activation of ERK1/2. Biochem Biophys Res Commun 2005 329:266-274.

21. Xu X, Yamamoto $H$, Sakon M, Yasui M, Ngan CY, Fukunaga H, Morita T, Ogawa M, Nagano H, Nakamori S, Sekimoto M, Matsuura N, Monden M: Overexpression of $\mathrm{CDC} 25 \mathrm{~A}$ phosphatase is associated with hypergrowth activity and poor prognosis of human hepatocellular carcinomas. Clin Cancer Res 2003, 9:1764-1772.

22. Ito Y, Yoshida H, Nakano K, Kobayashi K, Yokozawa T, Hirai K, Matsuzuka F, Matsuura N, Kakudo K, Kuma K, Miyauchi A: Expression of cdc25A and cdc25B proteins in thyroid neoplasms. Br J Cancer 2002, 86:1909-1913.

23. Ngan ES, Hashimoto Y, Ma ZQ, Tsai MJ, Tsai SY: Overexpression of Cdc25B, an androgen receptor coactivator, in prostate cancer. Oncogene 2003, 22:734-739.
24. Kudo Y, Yasui W, Ue T, Yamamoto S, Yokozaki H, Nikai H, Tahara E: Overexpression of cyclin-dependent kinase-activating CDC25B phosphatase in human gastric carcinomas. Jpn J Cancer Res 1997, 88:947-952.

25. Hernandez S, Bessa X, Bea S, Hernandez L, Nadal A, Mallofre C, Muntane J, Castells A, Fernandez PL, Cardesa A, Campo E: Differential expression of cdc25 cell-cycle-activating phosphatases in human colorectal carcinoma. Lab Invest 2001, 81:465-473.

26. Miyata H, Doki Y, Shiozaki H, Inoue M, Yano M, Fujiwara Y, Yamamoto H, Nishioka K, Kishi K, Monden M: CDC25B and p53 are independently implicated in radiation sensitivity for human esophageal cancers. Clin Cancer Res 2000, 6:4859-4865.

27. Guo J, Kleeff J, Li J, Ding J, Hammer J, Zhao Y, Giese T, Korc M, Buchler MW, Friess $\mathrm{H}$ : Expression and functional significance of $\mathrm{CDC} 25 \mathrm{~B}$ in human pancreatic ductal adenocarcinoma. Oncogene 2004, 23:71-81.

28. Dalal SN, Yaffe MB, DeCaprio JA: 14-3-3 family members act coordinately to regulate mitotic progression. Cell Cycle 2004, 3:672-677.

29. Mailand N, Podtelejnikov AV, Groth A, Mann M, Bartek J, Lukas J: Regulation of $\mathrm{G}(2) / \mathrm{M}$ events by $\mathrm{Cdc} 25 \mathrm{~A}$ through phosphorylationdependent modulation of its stability. EMBO J 2002, 21:5911-5920.

30. Bugler B, Quaranta M, Aressy B, Brezak MC, Prevost G, Ducommun B: Genotoxic-activated G2-M checkpoint exit is dependent on CDC25B phosphatase expression. Mol Cancer Ther 2006, 5:1446-1451.

31. Karlsson C, Katich S, Hagting A, Hoffmann I, Pines J: Cdc25B and Cdc25C differ markedly in their properties as initiators of mitosis. J Cell Biol 1999, 146:573-584.

32. Escalas N, Davezac N, De RJ, Baldin V, Mazars R, Ducommun B: Study of the cytolethal distending toxin-induced cell cycle arrest in HeLa cells: involvement of the CDC25 phosphatase. Exp Cell Res 2000, 257:206-212.

33. Bansal P, Lazo JS: Induction of Cdc $25 B$ regulates cell cycle resumption after genotoxic stress. Cancer Res 2007, 67:3356-3363.

34. Nagata A, Igarashi M, Jinno S, Suto K, Okayama H: An additional homolog of the fission yeast cdc25+ gene occurs in humans and is highly expressed in some cancer cells. New Biol 1991, 3:959-968.

35. Spitkovsky D, Jansen-Durr P, Karsenti E, Hoffman I: S-phase induction by adenovirus E1A requires activation of cdc25a tyrosine phosphatase. Oncogene 1996, 12:2549-2554.

36. Nakabayashi $\mathrm{H}$, Hara M, Shimizu K: Prognostic significance of CDC25B expression in gliomas. J Clin Pathol 2006, 59:725-728

37. Hu YC, Lam KY, Law S, Wong J, Srivastava G: Identification of differentially expressed genes in esophageal squamous cell carcinoma (ESCC) by CDNA expression array: overexpression of Fra-1, Neogenin, Id-1, and CDC25B genes in ESCC. Clin Cancer Res 2001, 7:2213-2221.

38. Bulavin DV, Higashimoto Y, Demidenko ZN, Meek S, Graves P, Phillips C, Zhao H, Moody SA, Appella E, Piwnica-Worms H, Fornace AJ Jr: Dual phosphorylation controls $\mathrm{Cdc} 25$ phosphatases and mitotic entry. Nat Cell Biol 2003, 5:545-551.

Pre-publication history

The pre-publication history for this paper can be accessed here: http://www.biomedcentral.com/1471-2407/10/233/prepub

doi: $10.1186 / 1471-2407-10-233$

Cite this article as: Wang et al., Overexpression of CDC25B, CDC25C and phospho-CDC25C (Ser216) in vulvar squamous cell carcinomas are associated with malignant features and aggressive cancer phenotypes BMC Cancer 2010, 10:233 